\title{
Cyclostationarity applied to acoustic emission and development of a new indicator for monitoring bearing defects
}

\author{
M. Kedadouche, M. Thomas a and A. Tahan \\ Department of Mechanical Engineering, École de Technologie Supérieure, 1100, Notre-Dame street West, Montreal, H3C 1K3, \\ Quebec, Canada
}

Received 17 June 2013, Accepted 15 May 2014

\begin{abstract}
The exploitation of cyclostationarity properties of vibratory signals is now more widely used for monitoring rotating machinery and especially for diagnosing bearing defects. The acoustic emission (AE) technology has also emerged as a reliable tool for preventive maintenance of rotating machines. In this study, we propose an experimental study that characterizes the cyclostationary aspect of acoustic emission (AE) signals recorded from a defective bearing ( $40 \mu \mathrm{m}$ on the outer race) to see its efficiency to detect a defect at its very early stage of degradation. An industrial sensor (UE10 000) is used. An electrical circuit converts the high frequency signal into an audible signal by heterodyning. The cyclic spectral density, which is a tool dedicated that put into evidence the presence of cyclostationarity, is used for characterizing the cyclostationary. Two new indicators based on this cyclostationary technique are proposed and compared for early detection of defective bearings.
\end{abstract}

Key words: Acoustic emission / cyclostationarity / indicator of cyclostationarity / rolling bearing

\section{Introduction}

Most researches on machinery fault diagnosis can be classified in time, frequency or time-frequency domain. In time domain, the RMS value of vibratory signals, Crest Factor (CF), Skewness and Kurtosis are the most used statistical descriptors since they are scalar $[1,2]$. Techniques advanced signal processing have been widely used in vibration. In the frequency domain, the envelope analysis, also known as amplitude demodulation, has been widely used and proved to be very effective in detecting bearing characteristic frequencies.

On the other hand, many research studies have been published on the detection and diagnosis of bearing defects by acoustic emission. Tandon and Choudhury [3] presented a detailed review of vibration and acoustic methods, noise measurements, shock waves and acoustic emission technique. They updated critical works [4] incorporating latest and advanced techniques. Yongyong et al. [5] presented a detailed review of the application of acoustic emission for monitoring bearings. There are few publications on the application of ultrasonic techniques for monitoring bearing condition $[6,7]$. Kim et al. [8] focused on the diagnosis of bearings (defect about $100 \mu \mathrm{m}$ ) operating at low speeds. Kedadouche et al. [9] presented a

a Corresponding author: marc.thomas@etsmtl.ca comparative study between acoustic emission and vibration. This study was focused on the diagnostic of bearing which presents a defect at an early stage $(40 \mu \mathrm{m})$. Chiementin et al. [10] used time domain indicators: RMS, Kurtosis, and proposed to improve the signal-to-noise ratio by applying denoising techniques (wavelet, spectral subtraction, sanc) on experimental acquired AE data. Liao et al. [11] used wavelet analysis and Zvokelj et al. [12] were interested in the application of empirical mode decomposition (EMD) applied to AE signals. Recently, Kilundu et al. [13] applied the cyclostationary on acoustic emission and showed the effectiveness of the spectral correlation and the ICS (integrated correlation spectral) indicator for monitoring bearing defect but with relatively big defect sizes.

The aim of this study is to investigate the effectiveness of acoustic emission techniques to detect bearing defects at a very early stage and compare the results with signals from vibration measurements, using an experimental approach. An artificially defect in the form of a scratch with a 40 microns size, induced on the outer ring of the bearing was used for this comparative study. It is the smallest bearing fault size that has ever been investigated in literature. The ultrasonic sensor (UE 10000) is used with an electrical circuit that converts the high frequency signal into an audible signal EA by heterodyning. The ultrasonic signals from a normal bearing and those of the damaged 


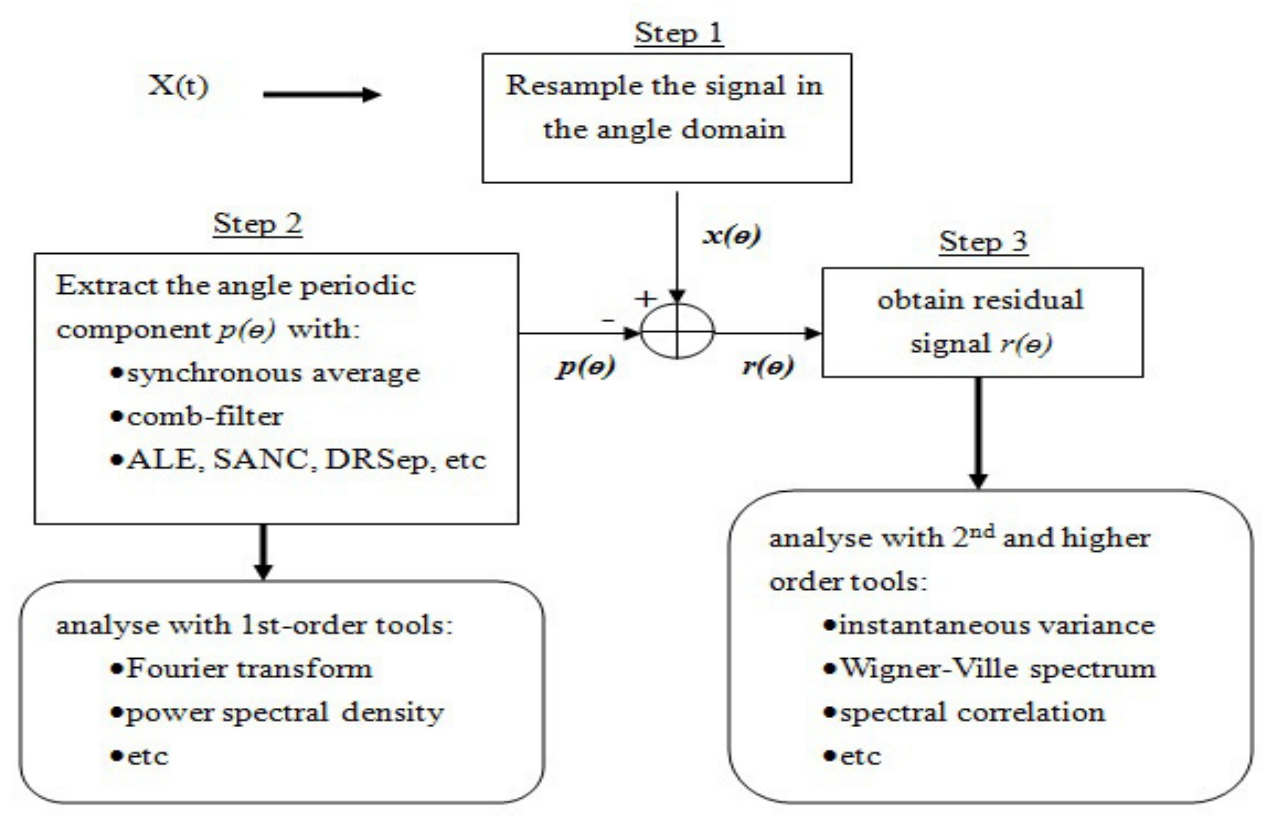

Fig. 1. Methodology for processing cyclostationary machine signals, Antoni et al. [15].

bearing are compared with vibration signals at speeds of rotation ranging from $300 \mathrm{rpm}$ to $900 \mathrm{rpm}$. This paper first highlights the cyclostationary character of AE associated with a defective bearing, and secondly, shows the effectiveness of the density spectral cyclic for diagnosis. Finally, new indicators for monitoring bearings based on cyclostationarity characteristics are presented.

\section{Cyclostationarity}

A signal is cyclostationary at an order " $n$ ", if its statistical properties, at an order " $n$ " are periodic. Antoni [14] presented a review of cyclostatinary processes and gives many examples. It is well known that the defects on the rolling bearing produce a series of shocks. These shocks are not perfectly periodic because of slips. Antony has suggested that these slips are non-stationary process and may be approximated as quasi-cyclostationary over only a limited period of time [15]. Kilundu et al. [13] note that the acoustic emission is similar to vibrations and suggest the same cyclostationarity properties for AE signal arising from a defective bearing. An efficient method for testing cyclostationarity of a signal $X(t)$ (residual part after extracting the deterministic part of the signal) is to compute the two-dimensional Fourier transform of its autocorrelation function $R_{x x}(t, \tau)$ i.e

$$
S_{x}(F, \alpha) \mathrm{d} f \mathrm{~d} x=D F T_{t \rightarrow \alpha, \tau \rightarrow f}\left\{R_{x x}(t, \tau)\right\}
$$

where $f$ and $\alpha$ are, respectively, the spectral and cyclic frequencies.

The cyclic power spectral density of the signal is estimated by using Welch's averaged periodogram method. The parameters and the method are set based on the recommendations of Antoni [21,22]. For this investigation the parameters employed for obtaining the averaged cyclic periodogram estimate were as follows:

- hanning window with $2 / 3$ overlap;

- window length: 256;

- cyclic frequency resolution: $0.2 \mathrm{~Hz}$.

\subsection{Methodologies for processing cyclostationary signals}

Antoni et al. [15] presented a review of some signal processing tools dedicated to cyclostationary signals (Fig. 1) when the signals are issued from rotating machines. Figure 1 presents an organization chart for different steps.

The technique consists in resampling the signal in the angle domain (Step 1) and extracting the angle periodic component using the synchronous average (Step 2). After that, the residual (2sd order) may be computed by extracting the periodic part (deterministic) from the original signal (Step 3). If we cannot resample the signal in the angle domain, other tools may be used which are dedicated to extract the deterministic part for any signal like Comb-filter, ALE, SANC and DRSep... etc. In the last decades, several blind algorithms were dedicated to the separation of mixtures of unobserved signals. Recently applied to mechanical systems and in particular to rotating machines that usually generate vibration signals exhibiting cyclostationarity, Bonnardot et al. [16] developed a multiple cyclic regression (MCR) technique for extracting the 2sd order of a signal. Boustany and Antoni [17] proposed a new method called SUBLEX which achieves to the same objectives under the same assumptions as [16], but using a different approach. After that, Boustany and 


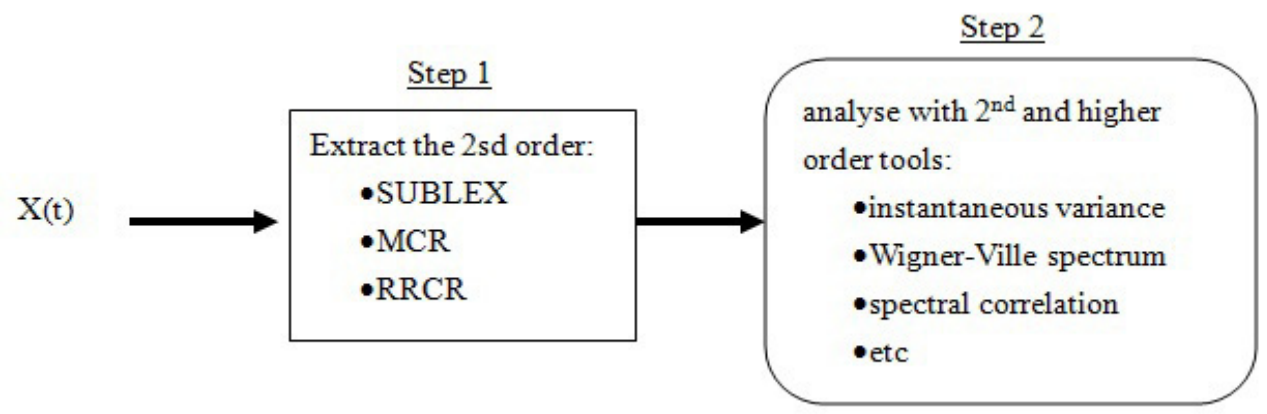

Fig. 2. Methodology for processing cyclostationary machine signals by extracting the second order.

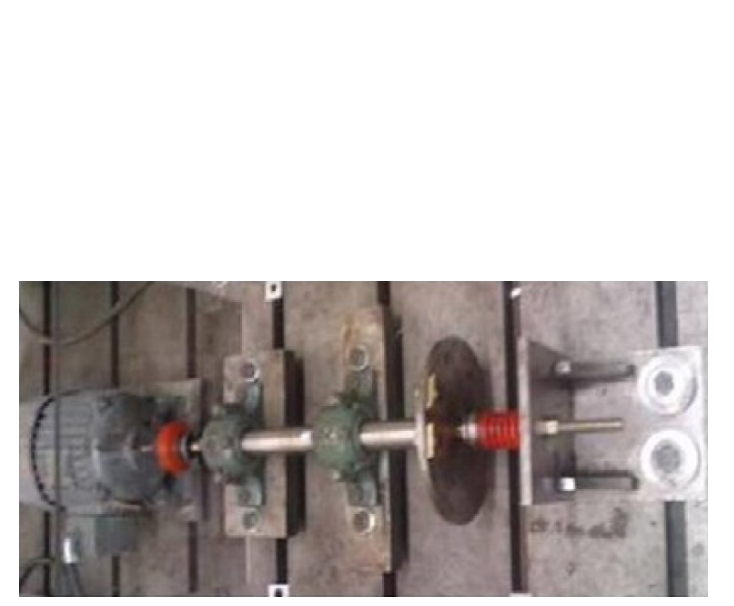

(a)

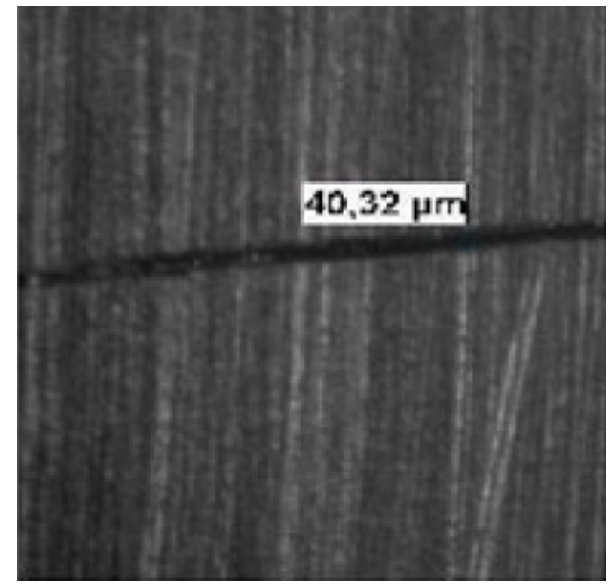

(b)

Fig. 3. (a) Experimental test bench, (b) the defect on outer ring.

Antoni [18] proposed a new effective method called RRCR (Reduced-Rank Cyclic Regression) that benefits from the respective advantages of the original MCR and SUBLEX methods but suppresses their drawbacks. All these technique may be used to study the cyclostationary of rotating machine (Fig. 2). In this paper, the last method (RRCR) is used for extracting the second order of the signal from the acoustic emission and from the vibration signal in order to compute their cyclic spectral density (CSD).

\section{Experimental study}

The test bench used in this study is shown in Figure 3a. A shaft is supported by two bearings and connected to a motor with a flanged coupling bolted rubber. Two systems were investigated, one with a healthy bearing (SKF, 1210 EKTN9) and the other with an artificially damaged bearing on the outer race (defect order: $\mathrm{BPFO}=7.24)$. The default size is 40 microns as shown in Figure $3 \mathrm{~b}$.

The equipments for vibration data collection and ultrasound are shown in Figure 4a. They consist in an accelerometer with a sensitivity of $100 \mathrm{mV} \cdot \mathrm{g}^{-1}$ and an ultrasound detector (UE Systems UltraProb 10 000). Both sensors are connected to an analogous digital converter (THOR Analyzer PRO: DT9837-13310) with a sampling frequency of $48 \mathrm{kHz}$. The latter is connected to a collectoranalyzer BETAVIB. The ultrasonic sensor used in this study operates in the lower ultrasonic spectrum from $20 \mathrm{kHz}$ to $100 \mathrm{kHz}$. A heterodyne circuit converts the high frequency acoustic emission signal as detected by the transducer around a central frequency Fc into an audible signal (0-7 kHz) (Fig. 4b). The heterodyned signal may then be recorded through conventional data acquisition system at $48 \mathrm{kHz}$. The mechanical system is excited by an unbalance mass rotating. The acoustic emission signals from the two bearings (healthy and faulty) are recorded at the central frequency of $30 \mathrm{kHz}$ which was found as the more sensitive to the defect when rotating at various speeds (300, 600 and $900 \mathrm{rpm})$.

\section{Results analysis and applications}

\subsection{Vibration signal}

Figures 5a and 5b show, respectively, the vibration signal of the damaged bearing when operating at $300 \mathrm{rpm}$ and its second order obtained by RCRR. Figure $5 \mathrm{c}$ shows 


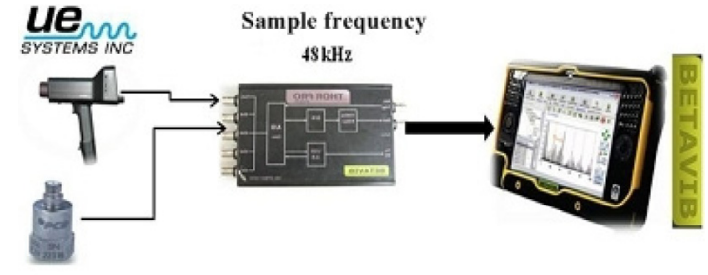

(a)

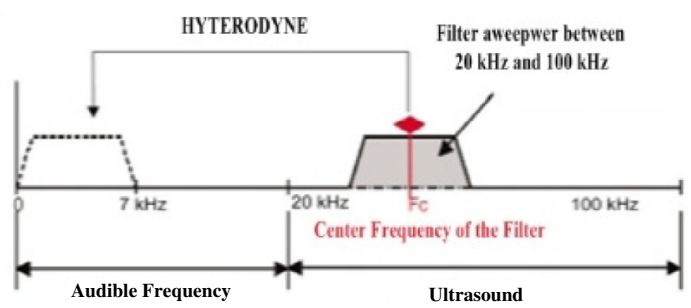

(b)

Fig. 4. (a) Data acquisition system, (b) heterodyne principle.

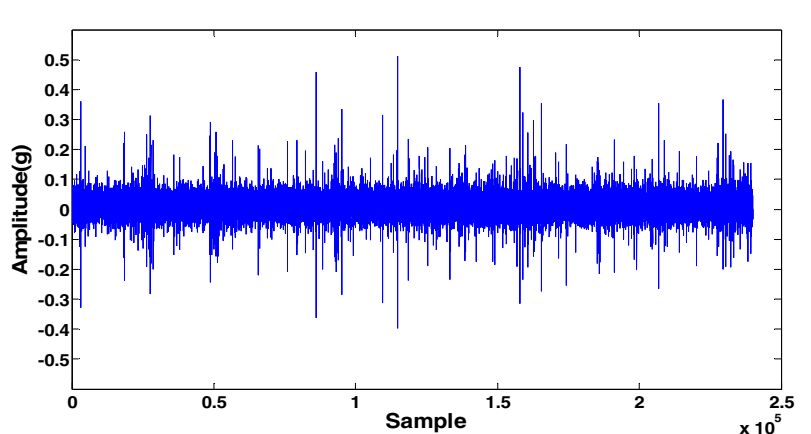

(a)

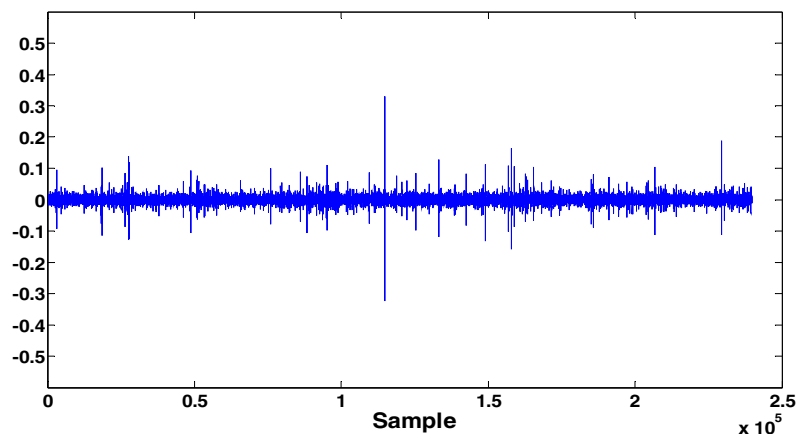

(b)

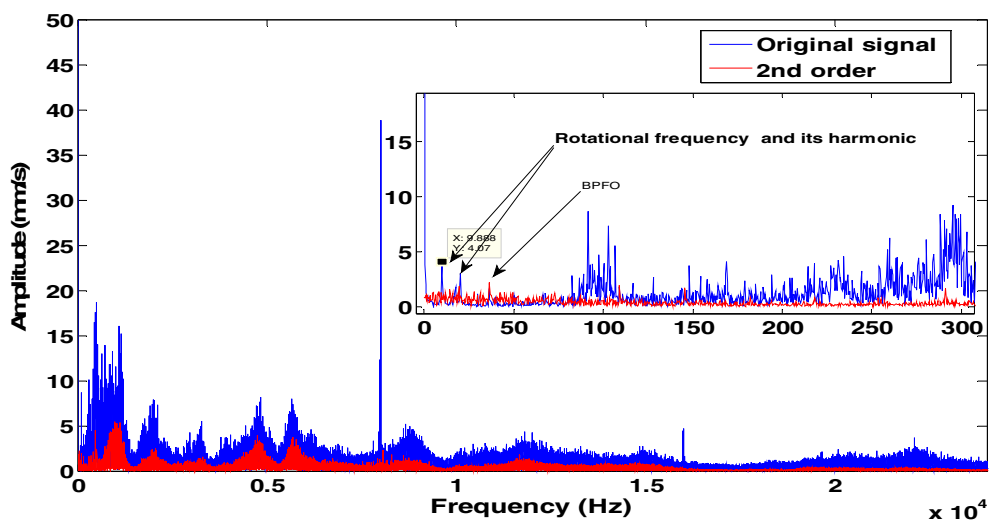

(c)

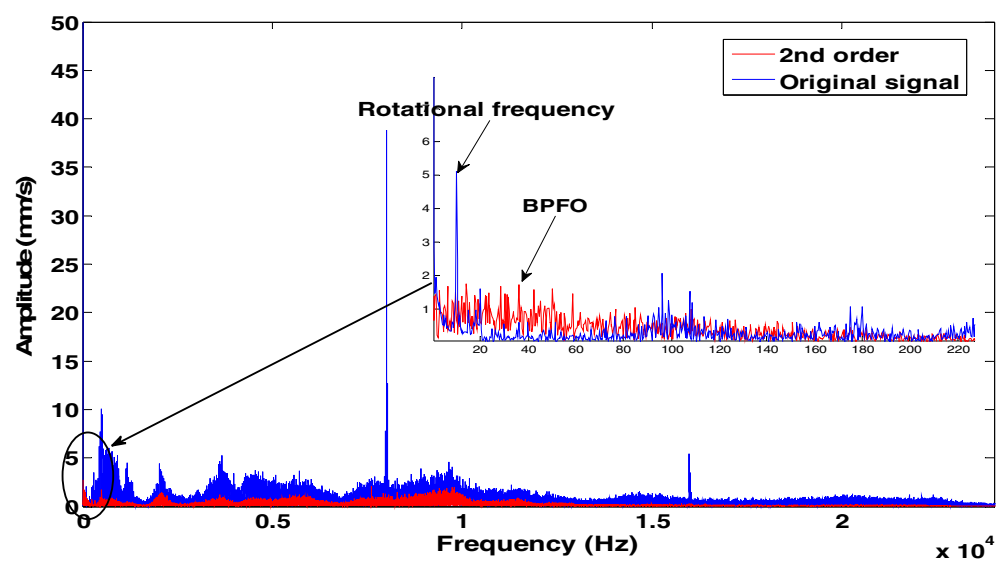

(d)

Fig. 5. (a) Vibration signal of the defective bearing, (b) second order after applying the RRCR method of the defective bearing, (c) spectrum of (a) and (b) of the defective bearing, (d) spectrum of the healthy bearing. 


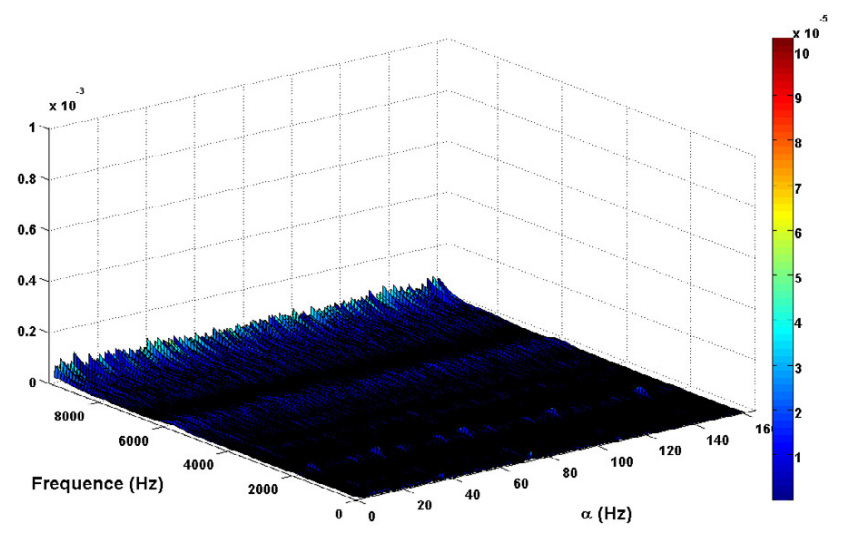

(a)

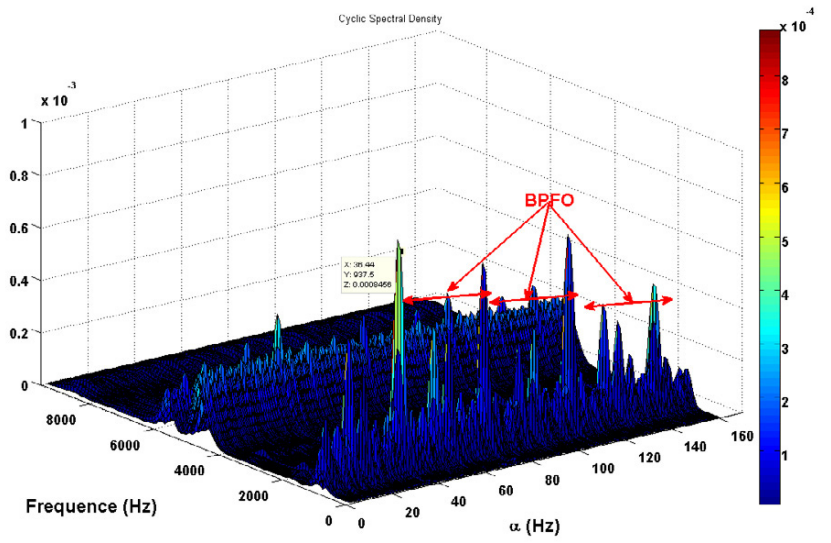

(b)

Fig. 6. CSD of vibration signal: (a) healthy bearing, (b) defective bearing.

the spectrum of the original signal (Blue line) and its 2nd order (Red line) for the defective bearing while Figure $5 \mathrm{~d}$ exhibits the spectrum for the healthy bearing. A zoom around $[0-300 \mathrm{~Hz}]$ reveals that the frequency related to the rotation of the shaft (deterministic part) exists only in the original signal, while we can observe the bearing frequency (BPFO) in the 2nd order spectrum in both cases (healthy and faulty), but with a very small amplitude. All other deterministic peaks were filtered in the 2nd order spectrum.

Figure 6 compares the cyclic spectral density (CSD) between the healthy and defective bearing. Figure $6 \mathrm{~b}$ clearly exhibits BPFO and its harmonics around the resonance of bearing which are located between [500-1500 Hz] and [4000-5000 Hz], with a level of energy definitely larger than for the healthy bearing (Fig. 6a).

\subsection{Acoustic emission signal}

Figures $7 \mathrm{a}$ and $7 \mathrm{~b}$ show, respectively, the ultrasonic signal of the damaged bearing when operating at $300 \mathrm{rpm}$ and its second order obtained by RCRR. We note that the second order is the same as the original signal when a defect is present. The acoustic emission doesn't detect information related to the rotational frequency (deterministic part), but we observe a decrease of noise. Figure $7 \mathrm{c}$ shows the spectrum of the original signal (Blue line) and its 2nd order (Red line) for the defective bearing while Figure $7 \mathrm{~d}$ exhibits the spectrum for the healthy bearing. A zoom around $[0-300 \mathrm{~Hz}]$ reveals that the spectrum of the $2 \mathrm{nd}$ order is the same as the spectrum of the original signal when a defect is present while its amplitude is lower when there is no defect. At this speed we can consider that the original signal recorded by acoustic emission is at order 2 . It is in fact the main advantage of acoustic emission measurements. However, the contribution of the deterministic part is lost.

Similarly to vibration analysis, Figure 8b clearly highlights the CSD of ultrasonic signal of the defective bearing, showing the manifestation of BPFO and its harmonics around the resonance $[500-1500 \mathrm{~Hz}]$ with a level of energy of the CSD definitely larger than for the healthy bearing (Fig. 8a).

\subsection{Comparison between vibration and acoustic emission}

Figures 9-11 represent the CSD of vibration and acoustic measurements for the defective bearing at speeds of $300 \mathrm{rpm}, 600 \mathrm{rpm}$ and $900 \mathrm{rpm}$, respectively. It can be noticed that acoustic emission detects BPFO and its harmonics for all speeds. However, the vibration measurements show only the manifestation of BPFO when operating at low speed (300 rpm) but only an increase of the energy at high frequencies around the resonance can be noticed for the two other speeds. In conclusion, it is clear that the acoustic emission measurement is more efficient to detect a defect than vibration measurements when the defect is very small $(40 \mu \mathrm{m})$ for an early stage of degradation when increasing the speed.

\subsection{New indicator of cyclostationary}

In order to track the defect evolution, Kilindu et al. [13] proposed a new indicator called Integrated Spectral Correlation denoted ICS, to evaluate energy at the defect frequency (Eq. (2)) from the CSD. The indicator is computed by integrating the magnitude of the spectral correlation between $\alpha_{1,2}=f_{\text {bearing }}(1 \pm 1 \%)$ along $\alpha$-axis, and for all the values along $f$-axis (Eq. (2)).

$$
\mathrm{ICS}=\sum_{\alpha=\alpha 1}^{\alpha 2} \sum_{0}^{f_{s} / 2}\left\|S_{x}^{\alpha}\right\|
$$

where $f_{\mathrm{s}}$ is the sample frequency. 


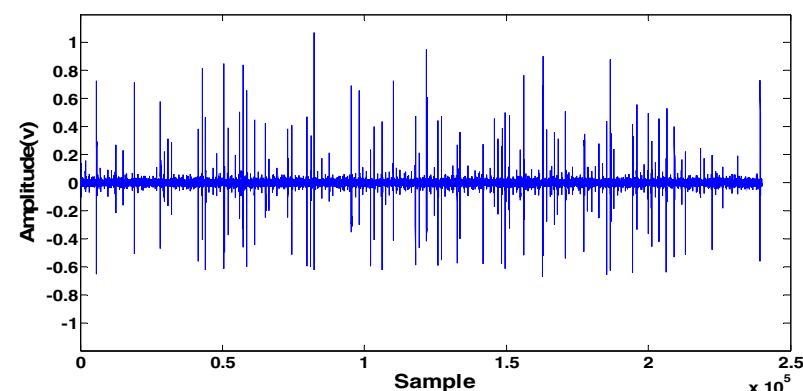

(a)

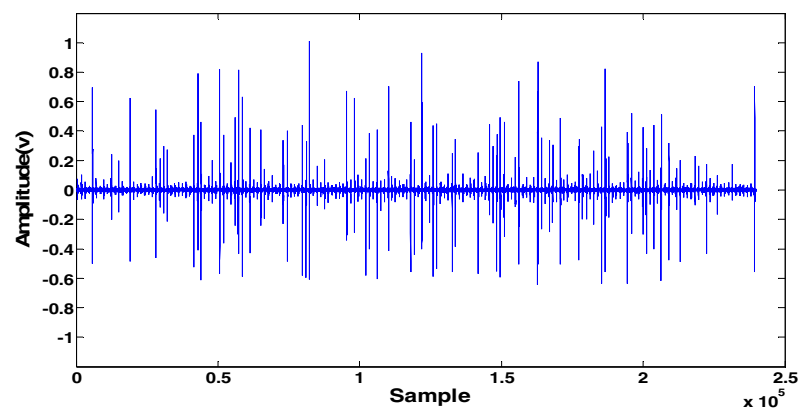

(b)

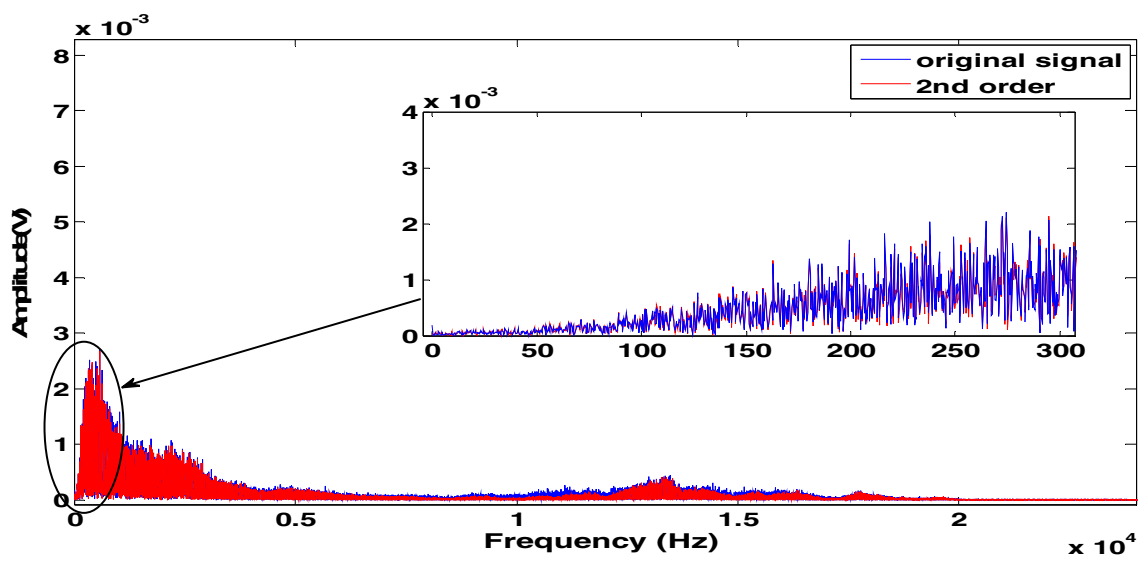

(c)

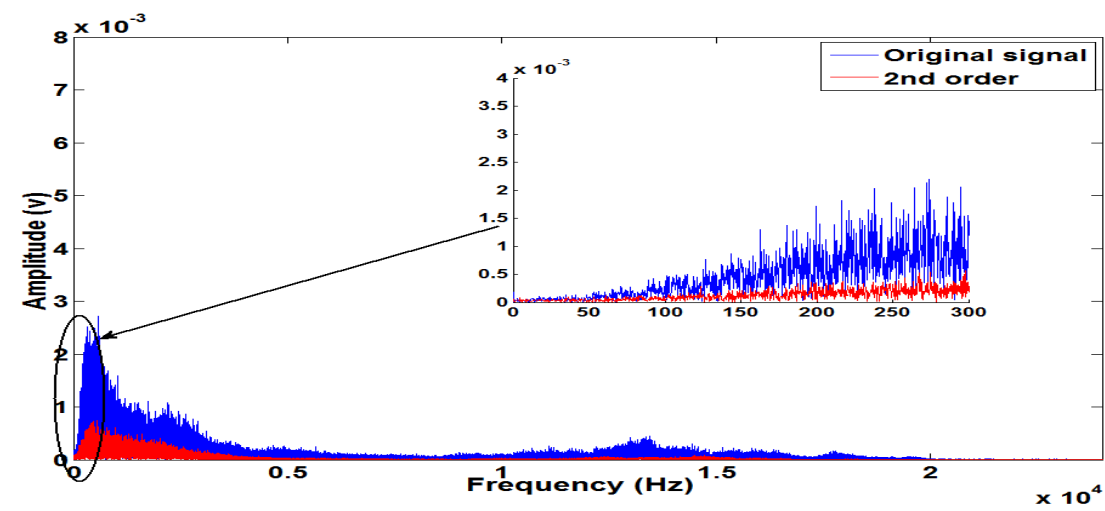

(d)

Fig. 7. (a) Ultrasonic signal of the defective bearing, (b) second order after applying the RRCR method of the defective bearing, (c) spectrum of (a) and (b) of the defective bearing, (d) spectrum of the healthy bearing.

If the defect is applied to outer race, the bearing frequency BPFO appears:

$$
f_{\text {bearing }}=\mathrm{BPFO}=\frac{N B}{2}\left(1-\frac{B d}{P d} \cos \theta\right) w
$$

where $B d$ is the diameter of the balls; $P d$ is the diametral pitch; $\theta$ is the contact angle; $N b$ is the number of balls and $w$ is the rotational speed.

However, it is well known that the increase of degradation of a faulty bearing not only causes an increase in the amplitude of the vibration frequencies of bearings, but also generates vibration harmonics of these frequencies as well as modulation frequencies. Consequently, the key of diagnostic relies on the number of harmonic of the bearing frequency and of their modulation frequencies $[19,20]$.

We have tried to develop two new indicators like ICS that are denoted $\mathrm{ICS}_{1}$ and $\mathrm{ICS}_{2}$ which take into account the amplitude modulation of the defects and the frequency resonance of the bearing:

- For the first one, the ICS is computed by integrating the magnitude of the cyclic spectral density between $(\mathrm{BPFO}-2.2 \times \mathrm{Fr}$ and $\mathrm{BPFO}+2.2 \times F r)$ along 


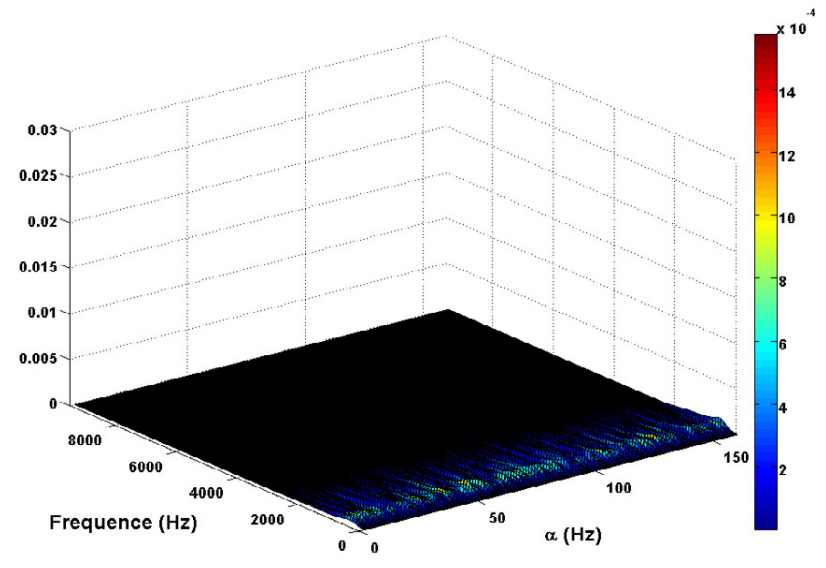

(a)

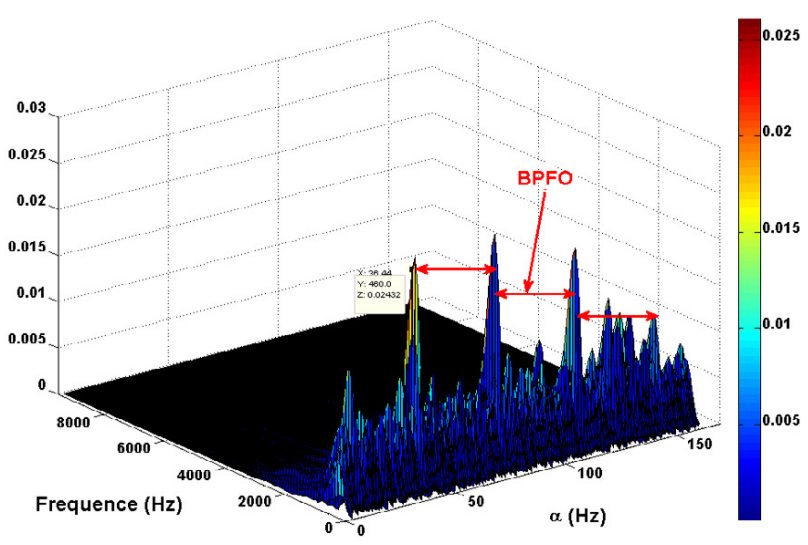

(b)

Fig. 8. CSD of ultrasonic signal: (a) healthy bearing, (b) defective bearing.

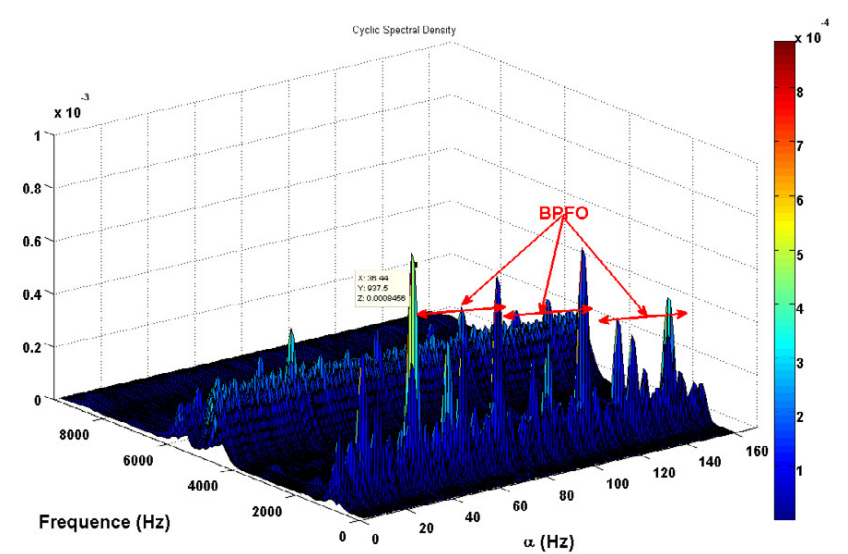

(a)

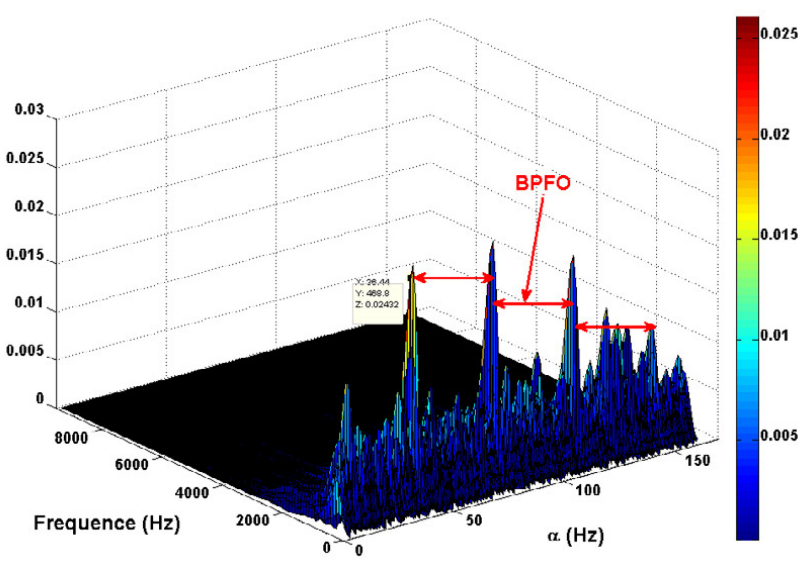

(b)

Fig. 9. CSD of defective bearing at speed of $300 \mathrm{rpm}$ : (a) vibration, (b) ultrasound.

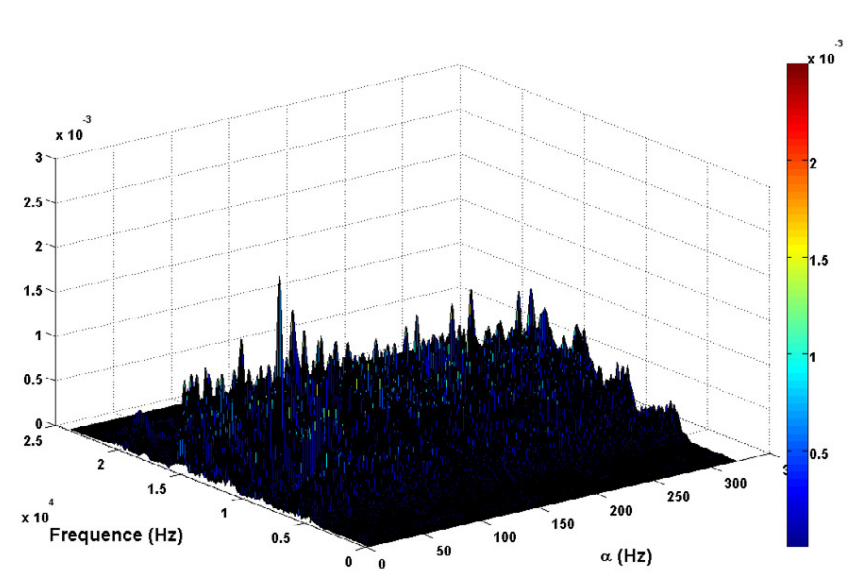

(a)

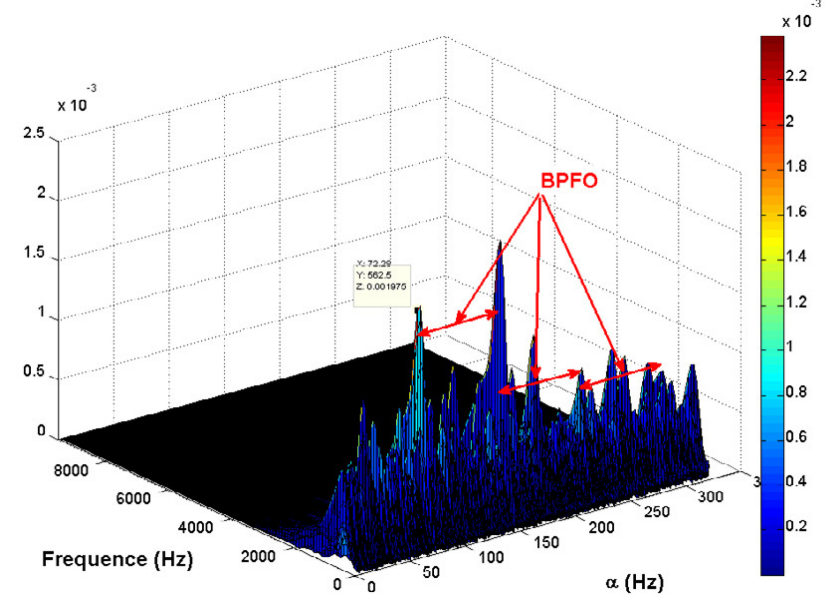

(b)

Fig. 10. CSD of defective bearing at speed $600 \mathrm{rpm}$ : (a) vibration, (b) ultrasound. 


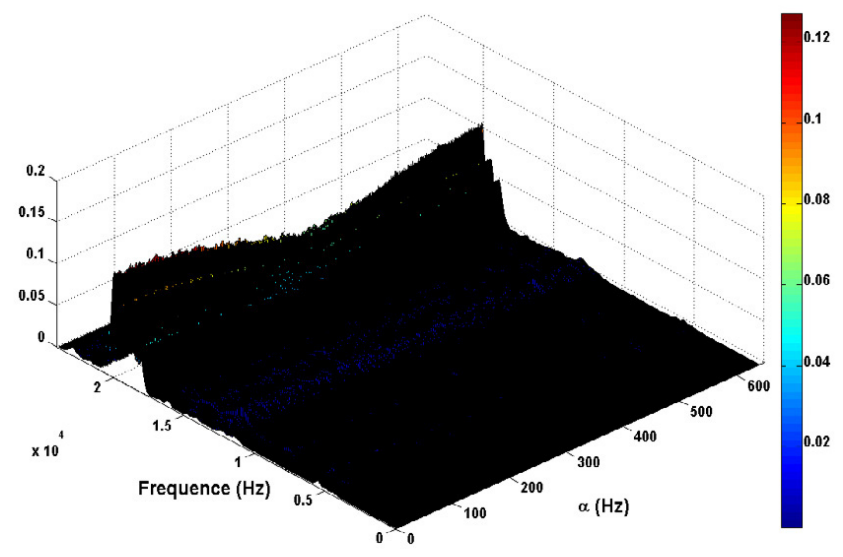

(a)

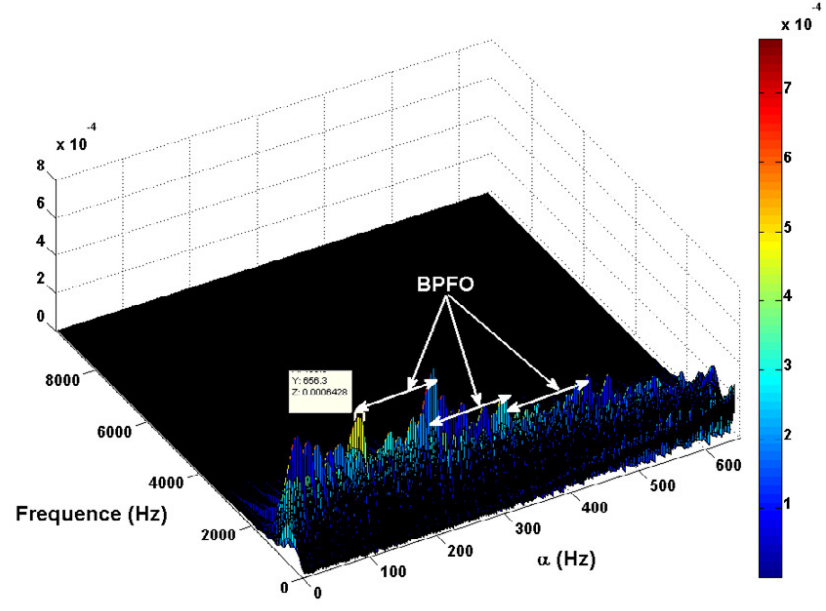

(b)

Fig. 11. SCD of defective bearing at speed $900 \mathrm{rpm}$ : (a) vibration, (b) ultrasound.
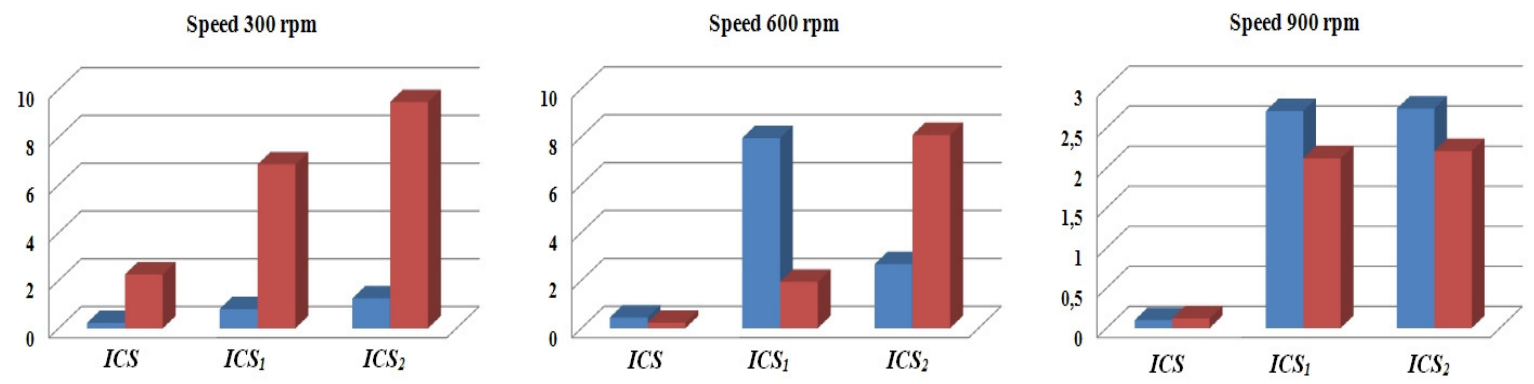

Fig. 12. Indicators of cyclostationarity at different speeds, healthy bearing, defective bearing.

$\alpha$-axis, and for all the values $f$ around the resonance (Eq. (4)).

$$
\mathrm{ICS}_{1}=\sum_{n=1}^{3} \sum_{a}^{b} \sum_{f 1}^{f 2}\left\|S_{x}^{\alpha}\right\|
$$

where $a=n \times \mathrm{BPFO}-\left(2.2 \times f_{\mathrm{r}}\right)$ and $b=n \times \mathrm{BPFO}+$ $\left(2.2 \times f_{\mathrm{r}}\right)$ and $f_{\mathrm{r}}$ is frequency of rotational speed.

- For the second one, the ICS is computed by integrating the magnitude of the cyclic spectral density between $(\mathrm{BPFO}-2.2 \times F r, \mathrm{BPFO}+2.2 \times F r)$ along $\alpha$-axis, and for all the values along $f$-axis (Eq. (5)).

$$
\mathrm{ICS}_{2}=\sum_{n=1}^{3} \sum_{a}^{b} \sum_{1}^{F_{s / 2}}\left\|S_{x}^{\alpha}\right\|
$$

Figure 12 shows the indicators (ICS, $\mathrm{ICS}_{1}$ and $\mathrm{ICS}_{2}$ ) computed for the healthy bearing (Blue bar) and the defective bearing (Red bar) at different speeds.

When rotating at $300 \mathrm{rpm}$, all the three indicators may distinguish between the healthy and defective bearing and it can be noticed that the two new indicators $\mathrm{ICS}_{1}$ and $\mathrm{ICS}_{2}$ ) are more sensitive than the ordinary ICS. However, at higher speeds (600 and $900 \mathrm{rpm}$ ), all these indicators are not efficient. We suppose that the problem may be due to the fact that when the defect size is very small, the amplitude of the healthy bearing is equivalent to the amplitude of the defective bearing due to the effect of other excitations that act in the high frequency domain (friction, lubricant, etc.).

For enhancing these indicators, we have investigated three new indicators. We propose to normalize the previous indicators by dividing them by time descriptors of the original signal. The new indicators (ICSN: Integrated Cyclic Spectral Normalised) are normalized relative to their RMS, Skewness, and kurtosis given by Equations (6)-(8).

$$
\begin{aligned}
\mathrm{SD} & =\sqrt{\frac{1}{N} \sum_{1}^{N}\left(x_{i}-\bar{x}\right)^{2}} \\
\text { Skewness } & =\frac{\frac{1}{N} \sum_{i=1}^{N}\left(x_{i}-\bar{x}\right)^{3}}{\left(\frac{1}{N} \sum_{i=1}^{N}\left(x_{i}-\bar{x}\right)^{2}\right)^{3 / 2}} \\
\text { kurtosis } & =\frac{\frac{1}{N} \sum_{i=1}^{N}\left(x_{i}-\bar{x}\right)^{4}}{\operatorname{RMS}^{4}}
\end{aligned}
$$


Table 1. Temporal descriptors for the healthy and the defective bearing at different speeds.

\begin{tabular}{cccccccc}
\hline \multicolumn{4}{c}{ Healthy bearing } & \multicolumn{3}{c}{ Defect bearing } \\
\hline Speed & Kurtoss & RMS & Skew & Speed & Kurtosis & RMS & Skew \\
\hline 300 & 153.169 & 0.021 & 2.073 & 300 & 118,05 & 0,038 & 0,93 \\
600 & 83.556 & 0.040 & 0.654 & 600 & 57.13 & 0.034 & 0.0057 \\
900 & 82.459 & 0.017 & 1.025 & 900 & 61.10 & 0.025 & 0.1355 \\
\hline
\end{tabular}
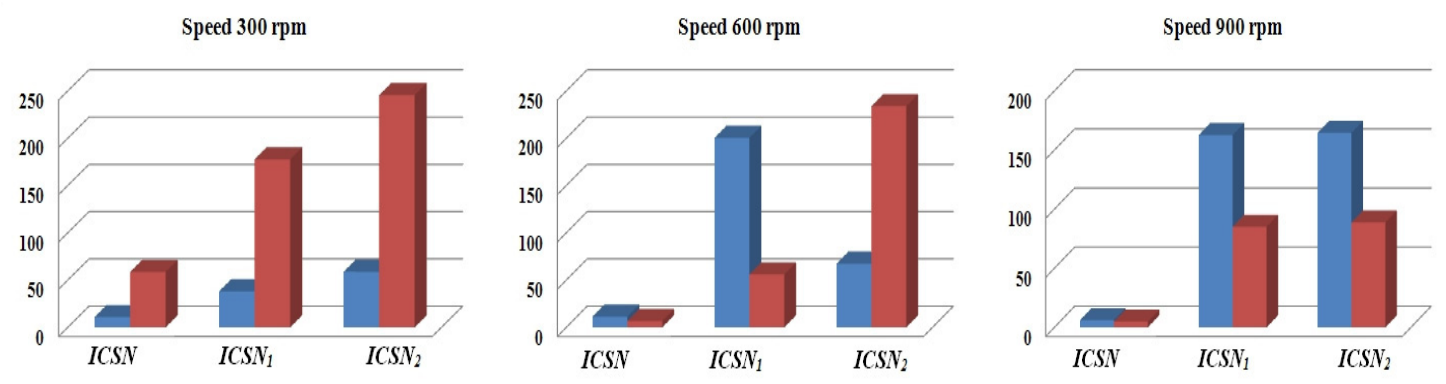

Fig. 13. Indicators of cyclostationarity normalized by RMS for different speeds, healthy bearing, defective bearing.
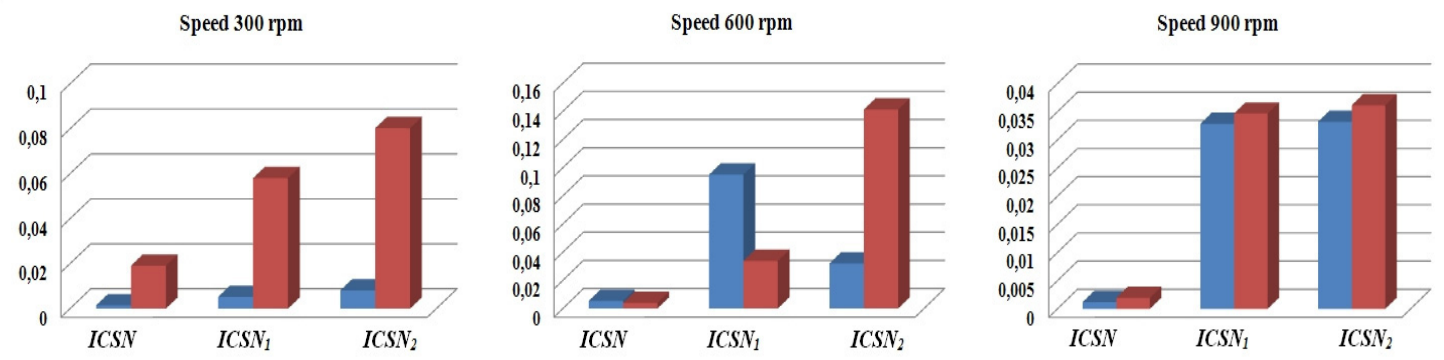

Fig. 14. Indicators of cyclostationarity normalized by Kurtosis for different speeds, healthy bearing, defective bearing.

Table 1 presents the values of the temporal descriptors of the healthy bearing and the defective one. It is well known that the Kurtosis and Skewness are so sensible to random chocks. Due to the effect of excitations, that act in the high frequency domain (friction, lubricant, etc.), the healthy bearing produces random chocks. For this reason we note that the Kurtosis and Skewness of the healthy bearing are greater than for the defective bearing.

Figures 13-15 show the indicators normalised by RMS, Kurtosis and Skewness, respectively, and computed for the healthy bearing (Blue bar) and the defective bearing (Red bar).

- When rotating at low speed $(300 \mathrm{rpm})$, all the indicators normalised are efficient and the $\mathrm{ICSN}_{2}$ is the best compared to others indicators.

- When rotating at $600 \mathrm{rpm}$, only $\mathrm{ICSN}_{2}$ makes a difference between the healthy and the defective bearing, for all the indicators.

- However, when rotating at higher speed (900 rpm), only $\mathrm{ICSN}_{2}$ normalised by Skewness (Fig. 15) allows for making a difference between the healthy and the defective bearing.

Figure 15 shows that all these indicators normalized by skewness were able to distinguish a defective bearing from a healthy one, whatever the rotating speed. However, the indicator $\mathrm{ICSN}_{2}$ normalised by Skewness revealed to be more sensitive than the two others by making a large difference between the healthy and the defective bearing for all the speeds (Fig. 15). Consequently, we propose to adopt this indicator $\left(\mathrm{ICSN}_{2}\right)$ for the monitoring of acoustic emission signals applied to bearing diagnosis.

\section{Conclusion}

This study focused on the potential of cyclostationary tools applied to the acoustic emission to detect a very small defect (40 microns) compared with the vibration measurement. Two bearings (defective and healthy) were investigated on a test bench at different speeds. This study highlights the cyclostationarity character of acoustic emission measurements. The cyclic spectral density (CSD) is computed for all acoustic emission and vibration signals and the results show that the acoustic emission measurement is more efficient than the vibration measurement for an early detection of degradation even when defect is very small $(40 \mu \mathrm{m})$ especially when increasing the speed. A new indicator (called $\mathrm{ICSN}_{2}$ ) is developed for the monitoring of acoustic emission signal. This indicator is based on the amplitude of the cyclic spectral density 

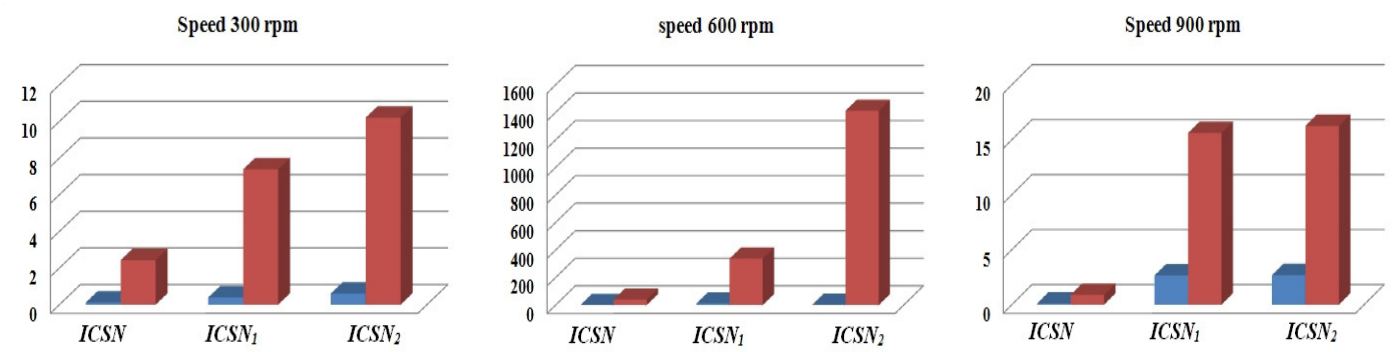

Fig. 15. Indicators of cyclostationarity normalized by Skewness for different speeds, healthy bearing, defective bearing.

computed into a bandwidth close to bearing frequencies and its harmonics and normalized by the Skewness of the time signal. It must be noticed that this study is the first one able to detect a so small defect on bearings (40 microns). Further tests are planned with several defects of different sizes to explore the full potential of this method; the results will be confirmed later.

Acknowledgements. The financial support of NSERC (Natural Sciences and Engineering Research Council of Canada), FQRNT (Fonds Québecois de la Recherche sur la Nature et les Technologies), MITACS Canada and Pratt \& Whitney Canada are gratefully acknowledged. The technical supports of R. Ginet and S. Koch from Pratt \& Whitney Canada were greatly appreciated.

\section{References}

[1] A.K.S. Jardine, D. Lin, D. Banjevic, A review on machinery diagnostics and prognostics implementing conditionbased maintenance, Mech. Syst. Signal Process 20 (2006) $1483-1510$

[2] S. Sassi, B. Badri, M. Thomas, Tracking surface degradation of ball bearings by means of new time domain scalar descriptors, Int. J. COMADEM 11 (2008) ISSN1363-7681 $36-45$

[3] N. Tandon, A. Choudhury, A review of vibration and acoustic measurement methods for the detection of defects in rolling element bearings, Tribol. Int. 32 (1999) 469-480

[4] A. Choudhury, N. Tandon, Application of acoustic emission technique for the detection of defects in rolling element bearings, Tribol. Int. 33 (2000) 39-45

[5] X.Z. Yongyong He, I. Michael, Friswell, Defect diagnosis for rolling element bearings using acoustic emission, J. Vib. Acoust. 131 (2009) (ASME)

[6] A. Dadouche, et al., Sensitivity of Air-Coupled Ultrasound and Eddy Current Sensors to Bearing Fault Detection, Tribol. Trans. 51 (2008) 310-323

[7] J. Shiroishi et al., Bearing condition diagnosis via vibration and acoustic emission measurements, Mech. Syst. Signal Process. 11 (1997) 693-705

[8] Y.-H. Kim et al., Condition Monitoring of Low Speed Bearings: A Comparative Study of the Ultrasound Technique Versus Vibration Measurements, in Engineering Asset Management, Springer London, 2006, pp. 182-191
[9] M. Kedadouche, M. Thomas, A. Tahan, Monitoring bearings by acoustic emission: a comparative study with vibration techniques for early detection, Proceedings of the 30th Seminar on machinery vibration, Canadian Machinery Vibration Association, Niagara Falls (ON, Canada), 2012, 17 p.

[10] X. Chiementin, D. Mba, B. Charnley, S. Lignon, J.-P. Dron, Effect of the denoising on acoustic emission signals, J. Vib. Acoust. 132 (2010)

[11] C. Liao, X. Li, D. Liu, Application of reassigned wavelet scalogram in feature extraction based on acoustic emission signal, J. Mech. Eng. 45 (2009) 273-279

[12] M. Zvokelj, S. Zupan, I. Prebil, Multivariate and multiscale monitoring of large-size low-speed bearings using ensemble empirical mode decomposition method combined with principal component analysis, Mech. Syst. Signal Process. (24) (2010) 1049-1067

[13] B. Kilundu, et al., Cyclostationarity of Acoustic Emissions (AE) for monitoring bearing defects, Mech. Syst. Signal Process. 25 (2011) 2061-2072

[14] J. Antoni, Cyclostationarity by examples, Mech. Syst. Signal Process. 23 (2009) 987-1036

[15] J. Antoni, F. Bonnardot, A. Raad, M. El Badaoui, Cyclostationary modelling of rotating machine vibration signals, Mech. Syst. Signal Process. (2004) 1285-1314

[16] F. Bonnardot, R.B. Randall, F. Guillet, Extraction of 2nd order cyclostationary sources-application to vibration analysis, Mech. Syst. System Process. 19 (2005) 1230-1244

[17] R. Boustany, J. Antoni, A subspace method for the blind extraction of a cyclostationary source: application to rolling element bearing diagnostics, Mech. Syst. Syst. Process. 19 (2005) 1245-1259

[18] R. Boustany, J. Antoni, Blind extraction of a cyclostationary signal using reduced-rank cyclic regressionA unifying approach, Mech. Syst. Syst. Process. (2008) $520-541$

[19] M. Thomas, J. Masounave, T.M. Dao, C.T. Le Dinh, F. Lafleur, Rolling element bearing degradation and vibration signature relationship, 2e Conférence Internationale sur les méthodes de surveillance et techniques de diagnostics acoustiques et vibratoires, SFM, Senlis, 1995, Vol. 1, pp. $267-277$

[20] J.I. Taylor, Identification of bearing defects by spectral analysis, J. Mech. Design 102 (1980)

[21] J. Antoni, Cyclic spectral analysis in practice, Mech. Syst. Signal Process. 21 (2007) 597-630

[22] J. Antoni, Cyclic spectral analysis of rolling element bearing signals: facts and fictions, J. Sound Vib. 304 (2007) $497-529$ 